\title{
METHODS OF SOLVING SOME PERSONNEL-CLASSIFICATION PROBLEMS*
}

\author{
D. F. Votaw, JR. \\ YALE UNIVERSTTY
}

The personnel-classification problems considered in this paper are related to those studied by Brogden (2), Lord (6), and Thorndike (8). Section 1 gives an approach to personnel classification. A basic problem and variations of it are treated in section 2; and the computation of a solution is illustrated in section 3. Two extensions of the basic problem are presented in section 4 . Most of the methods indicated for computing solutions are applications of the "simplex" method used in linear programming (see 1, Chs. XXII, XXIII). The capabilities of a high speed computer in regard to the simplex method are discussed briefly (see section 1).

1. Introduction. The type of problem to be considered is indicated by the illustration below. Consider two persons and two jobs, and suppose that the productivity of each person with regard to each job is known. Suppose further that each job must be filled and that each person must be assigned to one and only one job. It is assumed that the productivities can be represented as single numbers. For example, let them be as follows:

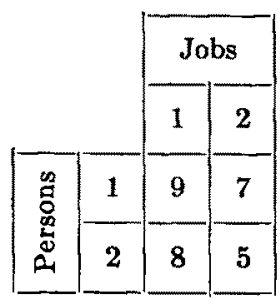

Thus 9 is the productivity of person 1 on job 1, 7 is the productivity of person 1 on job 2 , etc. To make maximum use of manpower the assigning agency would wish to establish an order of preference for the possible assignments and choose the assignment that heads the order. It would be natural to base such an ordering on average productivity. In the case at hand there are

*This work was supported in part by the United States Air Force under Contract AF 33(038)-25192, monitored by the Personnel Research Laboratory of the Air Training Command Human Resources Research Center. Permission is granted for reproduction, translation, publication, use, and disposal in whole or in part by or for the United States Government. 
two possible assignments. From (1:1) we obtain the following averages of the productivities associated with them:

Assignment

Person 1 on Job 1, Person 2 on Job 2 Person 1 on Job 2, Person 2 on Job 1
Average Productivity

$$
\begin{aligned}
& (9+5) / 2=7 \\
& (7+8) / 2=7.5
\end{aligned}
$$

It follows that the preferred assignment is formed by placing person 1 on job 2 and person 2 on job 1 . This gives the maximum possible average, which is 7.5. It should be noted that comparing assignments on the basis of the average productivity is equivalent to comparing them on the basis of the sum of productivities (see (1:2)).

The situation described above involving two persons and two jobs suggests the following general problem: Given $N$ persons, $N$ jobs, and the productivity of each person on each job; find an assignment of persons to jobs such that the average productivity is a maximum. (Recall that it is assumed that each job must be filled and that each person must be assigned to one and only one job.)* Problems similar to this have been considered by Brogden (2), Lord (6), and Thorndike (8, p. 217). A basic form of the problem and certain modifications of it are discussed in section 2. Section 3 gives an illustration of a step-by-step computation of a solution. Extensions of the basic problem are formulated in section 4.

Occasionally in personnel classification (e.g., in the military) there may be fewer job categories than jobs and/or fewer personnel categories than persons. This possibility has been taken into account in section 2 in the formulation of the basic problem. The $N$ persons are considered to be such that $a_{1}$ are alike, $a_{2}$ are alike, $\cdots, a_{m}$ are alike $\left(\sum_{i=1}^{m} a_{i}=N\right)$; thus there are only $m$ distinct personnel categories. Similarly, regarding the $N$ jobs it is assumed that $b_{1}$ are alike, $b_{2}$ are alike, $\cdots, b_{n}$ are alike $\left(\sum_{i-1}^{n} b_{i}=N\right)$; thus there are only $n$ distinct job categories. $\left(m=n=N=2\right.$ and $a_{1}=$ $a_{2}=b_{1}=b_{2}=1$ in the illustration associated with (1:1)). If, say, $x_{i i}$ persons of type $i$ are placed on jobs of type $j$, their contribution to the "group productivity" is assumed to be $c_{i i} x_{i i}$, where $c_{i i}$ denotes the productivity (or expected productivity) of the $i$ th type of person on the $j$ th type of job ( $i=1$, $\cdots, m ; j=1, \cdots, n)$. For a discussion of how the $c_{i j}$ 's might be determined see $(2,8)$; in some situations $c_{i}$ might be considered as a monetary saving.

Two extensions of the basic problem will be considered (see section 4). In one of them the number, $m$, of personnel categories is assumed to be indefinitely large, but the number, $n$, of job categories is assumed to be finite. Each personnel category is represented as a point in $n$-space, where the $n$ coordinates of the point represent the $n$ productivities associated with the category. An $n$-dimensional distribution is assumed to be associated with the

*In an actual situation the numbers of persons and jobs might be unequal; however, equality could be forced by introducing "dummy" jobs or persons. 
set of personnel categories. The essential characteristic of the second extension is that a vector $c_{1, i i}, \cdots, c_{k, i}$ replaces the quantity $c_{i j}$. This extension might be of interest when, say, skill and preference of each person regarding each job must be considered separately; e.g., $c_{1, i j}$ could represent skill and $c_{2, i}$ could represent preference (here $k=2$ ).

Most of the computational techniques suggested in this paper originated in linear programming. Hand computation of the solution of a general linear programming problem is illustrated in 1, Chapter XXII. It seems possible that an "analogy" machine for solving such problems could be developed (see 3, p. 73). The example in section 3 illustrates hand computation of a solution of the special linear programming problem referred to in section 2 (see also 1, Chapter XXIII). The National Bureau of Standards Eastern Automatic Computer (known as the SEAC) is coded for carrying out the simplex method used in the illustration. The SEAC is capable of solving a personnel-classification problem within a few hours when $m+n \leq 60$. It has obtained solutions of problems in which $m=4, n=9$, and $m=n=10$ in six minutes and twenty minutes, respectively. Government and military agencies have access to the SEAC through the National Bureau of Standards.

2. A Basic Problem in Personnel Classification. Suppose that with regard to $N$ persons there are $m$ mutually exclusive personnel categories, and that with regard to $N$ jobs there are $n$ mutually exclusive job categories. Let $a_{i}$ and $b_{i}$ be, respectively, the number of persons in the $i$ th category and the number of jobs in the $j$ th category $(i=1, \cdots, m ; j=1, \cdots, n)$. Assume that any two persons in the same category are essentially identical and that any two jobs in the same category are essentially identical. (This assumption involves no loss of generality since the case in which a job category or personnel category contains only one member is not excluded.) Let $c_{i i}$ be the productivity (or expected productivity) of any person in the $i$ th category on any job in the $j$ th category. Let $x_{i j}$ represent any number of persons in the $i$ th category who can be placed on jobs in the $j$ th category; the array $\left(x_{i}\right)$ is termed an "allocation." Let $T=\sum_{i, j} c_{i} x_{i j}$, which might be considered as the group productivity (or expected group productivity) associated with $\left(x_{i j}\right)$. It is required to find an allocation for which $T$ is maximized (see 8, p. 217). More specifically, the problem* is to find values $x_{11}^{(0)}, \cdots, x_{m n}^{(0)}$, of $x_{11}, \cdots, x_{m n}$, respectively, for which $T$ assumes its maximum value, subject to the conditions:

$$
\begin{aligned}
& \sum_{i} x_{i j}=b_{i}, \\
& \sum_{i} x_{i i}=a_{i}, \quad(i=1, \cdots, m ; j=1, \cdots, n),
\end{aligned}
$$

*In regard to this problem the author wishes to acknowledge valuable discussions with S. Kakutani, J. W. Tukey, M. A. Woodbury, and J. T. Dailey. 
[in (2:1) each $a_{i}, b_{i}$, and $x_{i}$ is a non-negative integer and $\sum_{i} a_{i}=\sum_{i} b_{i}=$ $N]$. This can be shown to be equivalent (except in minor respects) to the Hitchcock-Koopmans transportation problem, a special topic in linear programming, which can be solved by means of the simplex method (see 1, Chapter XXIII). Professor J. von Neumann has shown* that this problem is essentially the same as that of finding a best strategy in a certain zero-sum two-person game having a payoff matrix of order $(m+n) \times m n$. (See 7.)

An interesting special case of the problem is that in which each $c_{i j}$ has only two possible values, $\dagger$ say 1 and 0 (which, for example, could mean "qualified" and "not qualified," respectively). A variant of this special case is to determine whether $N$ is the maximum of $T$-and, if so, to find an allocation for which $T=N$. (If the maximum of $T$ is less than $N$, presumably the assigning agency would change one or more of the numbers $a_{1}, \cdots, a_{m}$, $b_{1}, \cdots, b_{n}$ or lower the standards regarding qualifications of persons for jobs.) The special case and this variant can be solved by means of Theorem 3 in (5).

A problem entirely similar to the one stated above (2:1) arises when $c_{i j}$ is considered not as a productivity but as the cost of training any person in the $i$ th category to do any job in the $j$ th category. In this situation $T$ represents the total cost of training, and one wishes to find an allocation for which $T$ assumes its minimum value.

When $N$ is large, one might wish to consider the numbers $a_{1}, \cdots, a_{m}$, $b_{1}, \cdots, b_{n}, x_{11}, \cdots, x_{m n}$ as population proportions; the conditions stated in (2:1) would then be replaced by the following conditions:

$$
\begin{gathered}
\sum_{i} x_{i i}=b_{i}, \\
\sum_{i} x_{i i}=a_{i}, \\
x_{i i} \geq 0,
\end{gathered}
$$

$\left(\sum_{i} a_{i}=\sum_{i} b_{i}=1 ; a_{i}, b_{i}>0 ; i=1, \cdots, m ; j=1, \cdots, n\right)$. The use of $(2: 2)$ in place of $(2: 1)$ introduces only minor modifications in the problems stated above.

3. An Illustrative Example. In this section a step-by-step procedure for solving problems of the type presented in section 2 will be illustrated. The procedure is an application of the simplex method (1, Chapter XXIII). Each step of the method is characterized by a distinct allocation. Before setting up the illustrative example we shall consider a special property of these allocations and indicate the underlying idea of the method.

A problem is said to be degenerate when a partial sum of $a$ 's equals a

*In a lecture at the Princeton University Game Seminar, October, 1951.

$\dagger$ In this case $m \leq 2^{n}$. 
partial sum of $b$ 's (the equality $\sum_{1}^{m} a_{i}=\sum_{1}^{n} b_{i}$ does not satisfy this condition). (A degenerate problem can be replaced by an essentially equivalent one that is non-degenerate.) In a non-degenerate problem each allocation used in the procedure has exactly $m+n-1$ positive $x_{i i}$ 's. We shall denote them by $x_{i_{i} i_{1}}, \cdots, x_{i_{i},}(r=m+n-1)$. With this set of $r$ positive $x_{i i}$ 's we associate quantities $u_{1}, \cdots, u_{m}, v_{1}, \cdots, v_{n}$, defined as follows:

$$
u_{i_{a}}+v_{i_{a}}=c_{i_{a} i_{a}} \quad(q=1, \cdots, r) .
$$

These equations can be solved for the $u$ 's and $v$ 's, where $u_{1}$, say, is set equal to 0 . Let $u_{i}+v_{i}=\bar{c}_{i j}$, say. The allocation is optimal (i.e., $\sum_{i,}, c_{i j} x_{i j}$ assumes its maximum value) if and only if

$$
\bar{c}_{i i} \geq c_{i i} \quad(\imath=1, \cdots, m ; \jmath=1, \cdots, n) .
$$

When the allocation associated with a given step is not optimal, one of its positive $x_{i i}$ 's can be "discarded" and a new positive $x_{i i}$ can be "introduced" to form an allocation for the next step. The new $x_{i j}$ can be chosen as one for which the corresponding $c_{i i}$ yields a largest value of $c_{i j}-\bar{c}_{i j}$. This choice determines which one of the positive $x_{i j}$ 's must be discarded. (See the discussion that follows the computing form below.) Every increase in $\sum_{i, i} c_{i j} x_{i i}$ from one step to the next is not less than a certain positive number, which depends on the $c_{i}$ 's, $a$ 's, and $b$ 's. Since the maximum sum is finite, the procedure involves at most a finite number of steps.

The simplex method will be used to solve the problem stated below. Consider a large group of persons and a correspondingly large group of jobs. Assume that there are exactly four types of persons and exactly three types of jobs; thus $m=4$ and $n=3$. Suppose that the productivities are as follows:

$$
\left(c_{i i}\right)=\left(\begin{array}{lll}
9 & 2 & 9 \\
1 & 8 & 8 \\
7 & 2 & 1 \\
9 & 8 & 0
\end{array}\right)
$$

( $c_{i j}$ is the actual or expected productivity of a person of type $i$ on a job of type $j$.) Let the proportions of the group associated with the four personnel categories be $a_{1}=.40, a_{2}=.20, a_{3}=.20, a_{4}=.20$; and let the proportions of the group required in the three job categories be $b_{1}=.35, b_{2}=.35, b_{3}=.30$. (Note that $\sum_{1}^{4} a_{i}=\sum_{1}^{3} b_{i}$ ) The problem is to find an allocation, $\left(x_{i 1}\right)$, for which the group productivity, $\sum_{i, i} c_{i i} x_{i}$, assumes its maximum value, where $\left(x_{i i}\right)$ is subject to the conditions given in (2:2). The allocation given in 
(3:7) is a solution.* The problem is non-degenerate since no partial sum of $a$ 's equals a partial sum of $b$ 's (i.e., it is not the case that an $a$, a sum of two $a$ 's, or a sum of three $a$ 's equals a $b$ or a sum of two $b$ 's).

All allocations used in the procedure have the property that exactly six of the $x_{i i}$ 's are positive. We may choose any such allocation as one with which to begin the procedure; accordingly, let the first one be as follows:

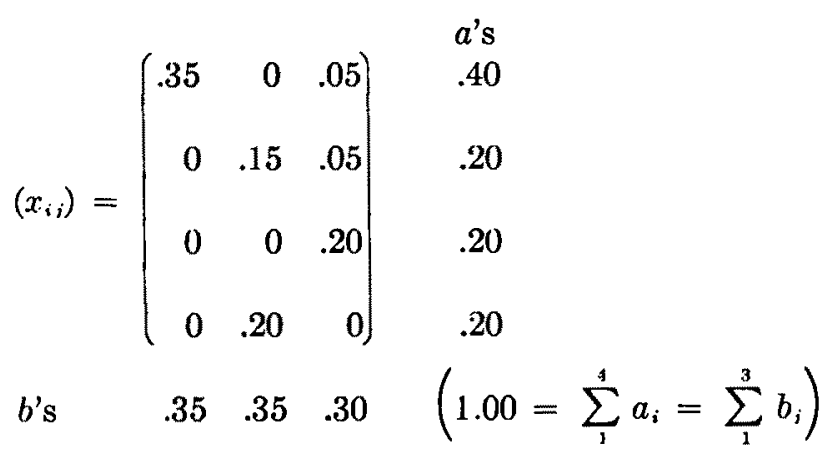

The six positive $x_{i j}$ 's in $(3: 4)$ can be considered as having been chosen in the following sequence: $x_{11}, x_{13}, x_{12}, x_{22}, x_{23}, x_{33}$. The way in which each was chosen can be described as follows: choose any $x_{i i}$, say $x_{11}$, and set $x_{11}$ equal to its maximum possible value, .35 , which is the minimum of $a_{1}=.40$ and $b_{1}=.35$ (this reduces the $4 \times 3$ array, $x_{i i}$, to a $4 \times 2$ array); next, in the reduced array choose any $x_{i j}$, say $x_{13}$, and set $x_{13}$ equal to its maximum possible value, .05 , which is the minimum of $a_{1}-x_{11}=.05$ and $b_{3}=.30$; continue this method of selection until the original array has been completely reduced.

We now introduce quantities $u_{1}, \cdots, u_{4}, v_{1}, \cdots, v_{3}$ which are to satisfy the following conditions [see (3:1)]:

$$
\begin{aligned}
& u_{1}+v_{1}=c_{11}, \\
& u_{1}+v_{3}=c_{13}, \\
& u_{4}+v_{2}=c_{42}, \\
& u_{2}+v_{2}=c_{22}, \\
& u_{2}+v_{3}=c_{23}, \\
& u_{3}+v_{3}=c_{33} .
\end{aligned}
$$

The six $c_{i j}$ 's in (3:5) correspond to the six positive $x_{i j}$ 's in (3:4). Solving (3:5) for $u_{2}, u_{3}, u_{4}, v_{1}, v_{2}, v_{3}$ we find that

*If $a_{1}, \cdots, a_{4}, b_{1}, \cdots, b_{8}$ had been replaced by $100 a_{1}, \cdots, 100 a_{4}, 100 b_{1}, \cdots, 100 b_{8}$, we could substitute the conditions in (2:1) for those in $(2: 2)$. A solution of this new problem would then be given by multiplying each $x_{i i}$ in $(3: 7)$ by 100 . 


$$
\begin{aligned}
& v_{1}=c_{11}-u_{1}, \\
& v_{2}=c_{22}-c_{23}+c_{13}-u_{1}, \\
& v_{3}=c_{13}-u_{1}, \\
& u_{2}=c_{23}-c_{13}+u_{1}, \\
& u_{3}=c_{33}-c_{13}+u_{1}, \\
& u_{4}=c_{42}-c_{22}+c_{23}-c_{13}+u_{1} .
\end{aligned}
$$

For example, from (3:5) we have that $v_{3}=c_{13}-u_{1}, u_{3}=c_{33}-v_{3}=c_{33}-$ $c_{13}+u_{1}$, etc. The allocation in (3:4) is optimal if and only if for every $(i, j) \bar{c}_{i j} \geq c_{i j}$, where $\bar{c} i j=u_{i}+v_{i}$ [see (3:2)]. It will be noted from Step 1 on the computing form below that this allocation is not optimal: e.g., $\bar{c}_{31}=$ $1<c_{31}=7$. This requires that a second step of the procedure be performed. The allocation associated with the second step will be formed from (3:4) by discarding $x_{33}$ and introducing $x_{31}$ [see the remarks immediately below $(3: 2)]$.

The information in $(3: 6)$ and the values of the $\bar{c}_{i j}$ 's are given in Step 1 of the computing form below. The first column of the form gives the positive $x_{i i}$ 's; the next column gives the values of the corresponding $c_{i j}$ 's. In the columns headed "Coefficients of $u_{1}, \cdots, v_{3}$ " the +'s and -'s given in (3:6) are indicated, where $u_{1}$ has been equated to 0 . Using the $c_{i j}$ column and the +'s and -'s in a $u$ or $v$ column, we can evaluate the $u$ or $v$ rapidly; e.g., $u_{4}=-9-8+8+8=-1$ (see the last row of the form for Step 1). The last column gives the products $c_{i} x_{i i}$ and their sum. The way of passing from one step to the next is indicated below in discussions of the steps. An interesting feature of the general procedure is that for any step each coefficient in the expression of a $u$ or $v$ as a linear combination of $c$ 's is $+1,0$, or -1 . The computing form is a slight modification of one given in (4).

Discussion of Step 1. It is not the case in Step 1 that each $\bar{c}_{i i} \geq c_{i}$; thus another step of the procedure must be performed. The maximum of the differences $c_{i j}-\bar{c}_{i j}$ is $c_{31}-\bar{c}_{31}=7-1=6$, and so $x_{31}$ will be given a positive value in the allocation for Step 2 (note that $\bar{c}_{31}$ has been italicized in Step 1). Since $\bar{c}_{31}=u_{2}+v_{1}$, we have that $\bar{c}_{31}=c_{11}+c_{33}-c_{13}$ (thus $\bar{c}_{31}+c_{13}=c_{11}+c_{33}$ ). This indicates that by adding, say, $d>0$ to $x_{31}$ and $x_{13}$ in (3:4) and subtracting $d$ from $x_{11}$ and $x_{33}$ a new allocation will be formed which differs from (3:4) in that the new $x_{31}$ will be positive and, if $d$ is sufficiently large, the new $x_{11}$ or $x_{33}$ will equal 0 . Since in Step $1 x_{33}=.20$ is smaller than $x_{11}=.35$, we set $d$ equal to .20 (thus the new $x_{33}$ will be 0 ). It follows from the expression above for $\bar{c}_{31}$ that $c_{33}=\bar{c}_{31}+c_{13}-c_{11}$; from this we can easily determine the coefficients of the $u$ 's and $v$ 's for Step 2; e.g., in Step $1 u_{3}=c_{33}-c_{13}$, thus in Step $2 u_{3}=c_{31}+c_{13}-c_{11}-c_{13}=c_{31}-c_{11}$.

Discussion of Step 2. The $\bar{c}_{i}$ 's obtained in Step 2 are not such that each 


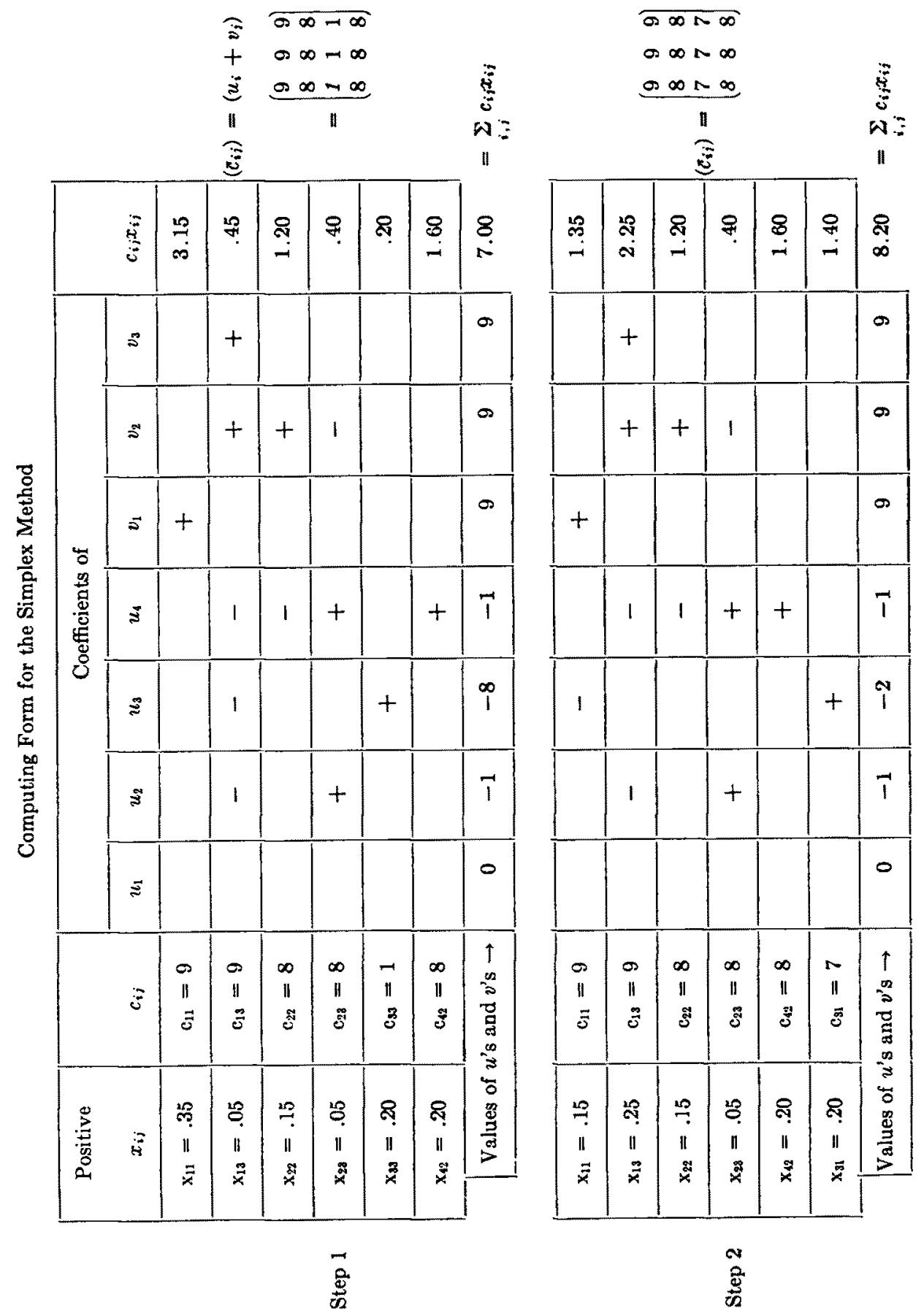


D. F. VOTAW, JR.

$\cos 10$

$\underbrace{\infty \infty \infty \infty}_{11} \frac{\pi}{4}$
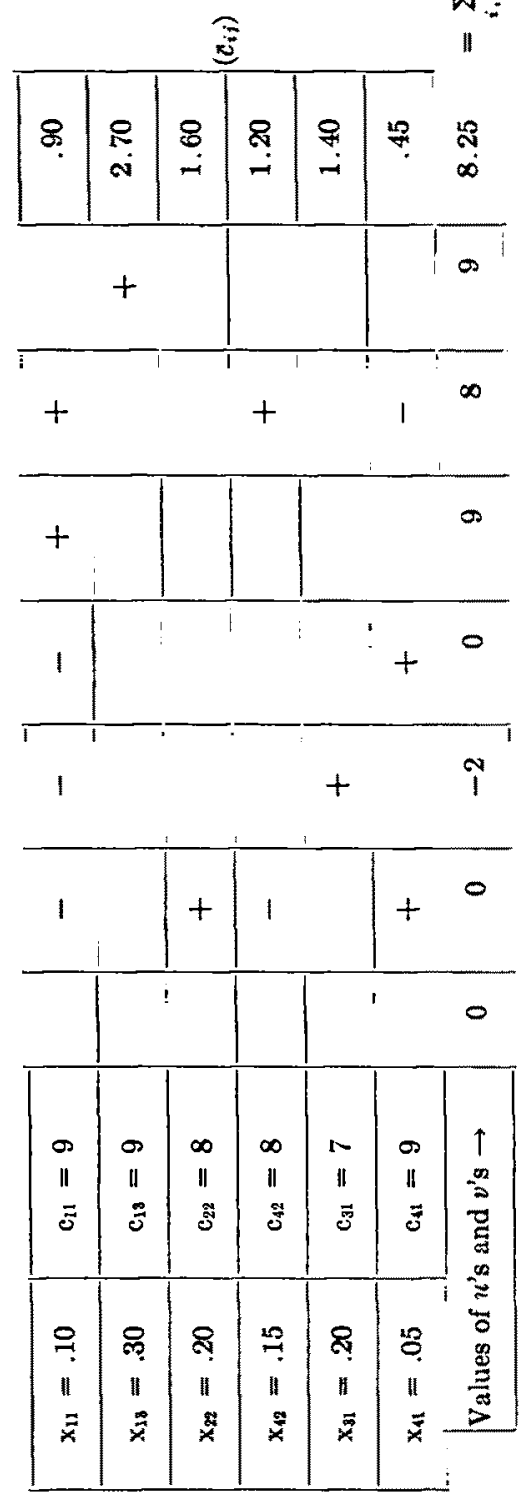

$\frac{0}{9}$ 
$\bar{c}_{i j} \geq c_{i j}$; hence a third step of the procedure must be performed. There is one (and only one) case in which $c_{i j}-\bar{c}_{i i}$ is positive-namely, $c_{41}-\bar{c}_{41}=$ $9-8=1$; thus $x_{41}$ should be positive in the new allocation. (Note that $\bar{c}_{41}$ is italicized in Step 2.) Since $\bar{c}_{41}+c_{13}+c_{22}=c_{11}+c_{23}+c_{42}$, it follows that .05 can be added to $x_{41}, x_{13}$, and $x_{22}$ and subtracted from $x_{11}, x_{23}$, and $x_{42}\left(x_{23}\right.$ 's value becomes 0$)$. It should be noted that $c_{23}=\bar{c}_{41}+c_{13}+c_{22}-$ $c_{11}-c_{42}$; by means of this we can easily determine coefficients of the $u$ 's and $v$ 's for Step 3.

Discussion of Step 3. The $c_{i i}$ 's associated with Step 3 are such that each $\bar{c}_{i j} \geq c_{i ;}$; thus the procedure ends with this step, and the allocation involved is a solution of the problem. The sum (8.25) obtained is at least as large as the sum associated with any other allocation. From the first column of Step 3 we have that the solution, $\left(x_{i i}\right)$, is as follows:

$$
\left(x_{i j}\right)=\left(\begin{array}{rrr}
.10 & 0 & .30 \\
0 & .20 & 0 \\
.20 & 0 & 0 \\
.05 & .15 & 0
\end{array}\right) .
$$

An optimal classification of the group of persons is formed when the persons of type $i$ assigned to jobs of type $j$ constitute a proportion $x_{i j}$ of the total group, where $x_{i i}$ is given in (3:7) $(i=1, \cdots, 4 ; j=1, \cdots, 3)$. The average productivity associated with this optimal classification is 8.25 .

4. Extensions of the Problem. Two different extensions will be presented. The first involves a population of personnel categories with which an $n$ dimensional distribution is associated; the second involves replacement of the scalar, $c_{i}$, by a vector.

Let $S$ be an $n$-space, and represent any point in $S$ by $\left(z_{1}, \cdots, z_{n}\right)$. Represent the $i$ th row of $\left(c_{i j}\right)$ as a point, $\left(c_{i 1}, \cdots, c_{i n}\right)$, in $S(i=1, \cdots, m)$ and let $F_{m}\left(z_{1}, \cdots, z_{n}\right)$ be the distribution function associated with the $m$ points representing the $m$ rows of $\left(c_{v i}\right)$; thus, in the problem associated with $(2: 2)$

$$
F_{m}\left(z_{1}, \cdots, z_{n}\right)=\sum_{a} a_{0},
$$

where $q$ ranges over all values of $i$ such that for every $j, c_{i j} \leq z_{j} . F_{m}\left(z_{1}\right.$, $\left.\cdots, z_{n}\right)$ is the probability that the $n$ productivities, say $c_{1}, \cdots, c_{n}$, of an individual selected at random would be such that $c_{i} \leq z_{i}(j=1, \cdots, n)$. $F_{m}\left(z_{1}, \cdots, z_{n}\right)$ is a discontinuous distribution; an extension of the problem arises when the population is such that the distribution, say $F\left(z_{1}, \cdots, z_{n}\right)$, is continuous (e.g., a cumulative normal $n$-variate distribution). This extension is considered in (2), (6), and (8). The problem is to find $n$ mutually ex- 
clusive regions, say $R_{1}, \cdots, R_{n}$, in $S$ such that $\sum_{i} \int_{R_{i}} z_{1} d F$ is maximized subject to the restrictions:

$$
\int_{R_{i}} d F=b_{i}, \quad\left(b_{i} \geq 0 ; \sum_{i} b_{i}=1 ; j=1, \cdots, n\right) .
$$

The optimal classification of the population would consist in assigning all individuals in $R_{j}$ to jobs in the $j$ th category $(j=1, \cdots, n)$. It follows from (6) that the regions would be determined by solving $n$ simultaneous equations in $n$ unknowns. A solution can be obtained very easily when $n=2$ (e.g., see 8, pp. 218-219). An approximate solution would be obtained by "grouping"--i.e., by approximating $F\left(z_{1}, \cdots, z_{n}\right)$ by a discontinuous distribution, say $F_{m}\left(z_{1}, \cdots, z_{n}\right)$-and then solving a problem of the type associated with (2:2). The quantity $a_{i}$ in (2:2) would represent the amount of a "jump" in the approximating distribution, $F_{m}\left(z_{1}, \cdots, z_{n}\right)(i=1, \cdots, m)$.

In the first problem stated in section 2, let $N$ be large and let $c_{i j}$ be replaced by a vector $\left(c_{1, i}, \cdots, c_{k, i}\right)(i=1, \cdots, m ; j=1, \cdots, n)$. (See the remarks in the next-to-last paragraph of section 1 regarding a case where $k$ would equal 2.) Let $T_{h}=\sum_{i, i} c_{h, i} x_{i j}(h=1, \cdots, k)$; and let $R=\sum_{h} g_{h} T_{h}$ be a composite indicator of the value of an allocation $\left(x_{i j}\right)\left(g_{1}, \cdots, g_{k}\right.$ are weights). Note that $R=\sum_{i, i} w_{i i} x_{i i}$, where $w_{i j}=\sum_{b} g_{h} c_{h, i i} ; w_{i i}$ is like the $c_{i j}$ in section 2. The problem is as follows: Find an allocation for which $R$ assumes its maximum value, where $x_{11}, \cdots, x_{m n}, a_{1}, \cdots, a_{m}, b_{1}, \cdots, b_{n}$ are subject to $(2: 2)$ and to inequalities of the form $T_{h} \geq e_{h}$ (here $e_{h}$ represent a minimum acceptable value of $T_{h}$; however, the problem is essentially unchanged if for any $h$ the inequality is reversed). A preliminary is to determine whether there is any allocation satisfying the specified conditions.

The problem stated above is an example of a linear programming problem, which can be solved by means of the simplex method (see 1, Chapters XX, XXI, XXII).

\section{REFERENCES}

1. Activity analysis of production and allocation (T. C. Koopmans, Editor). Cowles Commission Monograph No. 13. John Wiley and Sons, New York, 1951.

2. Brogden, Hubert E. An approach to the problem of differential prediction. Psychometrika, 1946, 11, 139-154.

3. Brown, G. W., and von Neumann, J. Solutions of games by differential equations in Contributions to the theory of games, Annals of Mathematics Study No. 24, edited by H. W. Kuhn and A. W. Tucker. Princeton: Princeton Univ. Press, 1950.

4. Dantzig, George B. Simplex solution of the Hitchcock-Koopmans transportation problem, Headquarters USAF Comptroller, February, 1950 (revised).

5. Hall, Philip. On representatives of subsets. J. London math. Soc., 1935, 10, 26-30.

6. Lord, Frederic M. Notes on a problem of multiple classification. Psychometrika, $1952,17,297-304$.

7. von Neumann, J. and Morgenstern, O. Theory of games and economic behavior, 2nd ed. Princeton: Princeton Univ. Press, 1947. 
8. Thorndike, Robert L. The problem of elassification of personnel. Psychometrika, $1950,15,215-235$.

Manuscript received 11/1/51

Revised manuscript received $\$ / 9 / 58$

Addition at proof reading: The simplex method provides a general solution of the basic problem stated in section 2; however, it may be awkward when $m$ or $n$ is large (see section 3). P. S. Dwyer has proposed some methods including a modification of the simplex method. T. E. Easterfield's paper (A combinatorial algorithm, J. Lond. Math. Soc., 1946, 21, 219-226) gives another interesting method. Procedures for obtaining approximate solutions are given in section III of a forthcoming bulletin by D. F. Votaw, Jr., and J. T. Dailey (Assignment of Personnel to Jobs, Human Resources Research Center, Lackland Air Force Base, San Antonio, Texas). 